\title{
Abhandlung
}

Peter A. Miglus*

\section{Ein Felsrelief in der Schlucht Darband-i Ramkan nahe Rania und die Geschichte seiner Erforschung}

DOI 10.1515/za-2016-0007

\begin{abstract}
Der vorliegende Aufsatz beschreibt die Erforschung eines wenig bekannten Felsreliefs, das in der Schlucht Darband-i Ramkan am östlichen Rand der Rania-Ebene über dem Kleinen Zab angebracht wurde. Bei diesem stark verwitterten Bild handelt es sich um die Darstellung eines Triumphators über einem besiegten Feind, die vermutlich im 19.-18. Jahrhundert v. Chr. entstanden ist.
\end{abstract}

Felsreliefs des ausgehenden dritten und beginnenden zweiten vorchristlichen Jahrtausends im westlichen Zagros-Gebirge gehören zu den ältesten Denkmälern dieser Art. Herrscherbilder von Sar-i Pol-i Zohab an der ZagrosPforte und die weiter nördlich angebrachten Reliefs von Darband-i Belula (Šeh Han/Hurin), Darband-i Gaur und Bitwāta wurden bereits umfassend publiziert. Fragen zu ihrer Datierung und Deutung sorgten für lebhafte Diskussionen, ${ }^{1}$ weniger beachtet wurde hingegen das über dem Kleinen Zab eingemeißelte Relief von Darband-i Ramkan. Im Standardwerk von Jutta Börker-Klähn (1982, $140 \mathrm{f}$. Nr. N 34) über die altvorderasiatischen Stelen und Felsreliefs wurde es kurz beschrieben und interpretiert; Eva Braun-Holzinger (2007, 149-156), die den Herrscherbildern aus dem Zagros einige Seiten widmete, erwähnte es gar nicht; zuletzt handelte es Simone Mühl (2013, $145 \mathrm{f}$. Anm. 890) in einer Fußnote ab. Die knapp gehaltenen Angaben zum Inhalt des Bildes sind mit seinem schlechten Erhaltungszustand zu erklären, teilweise aber auch mit verwirrenden Berichten seiner Besucher.

Das Relief befindet sich am Austritt des Kleinen Zab aus den Bergmassiven Asos und Kēwarhesh in die Rania-Ebene (Abb. 1-2). ${ }^{2}$ Die Schlucht ist unter den Namen

1 Die meiste Literatur ist bei Börker-Klähn (1982) zu finden. Darüber hinaus s. vor allem Postgate/Roaf (1997); Shaffer/Wasserman/Seidl (2003), Mofidi Nasrabadi (2004).

2 S. die Karte von Edmonds (1957, nach S. 262; hier Abb. 1). Vgl. Beschreibung von H. Ingholt bei Mortensen (1970, 11); dort ist der südöstliche Massiv als „Agos“ bezeichnet. Levine (1974, 6 fig. 6, 9), nennt die Berge am Darband-i Ramkan Kuh-i Resh und Kurkur Dagh.

* Corresponding author: Peter A. Miglus, Institut für Ur- und Frühgeschichte und Vorderasiatische Archäologie, Universität Heidelberg, E-mail: peter.miglus@uni-heidelberg.de
Darband-i Ramkan oder Sungasur Gorge und heute auch Darband-i Rania bekannt (Abb. 3). Sie bildet den Hauptzugang zur Rania-Ebene von Osten, ${ }^{3}$ und ihre strategische Bedeutung wird unter anderem durch mehrere Befestigungsanlagen belegt, welche die Schlucht von der südlichen Seite flankieren. ${ }^{4}$ Das Bild wurde auf einer zerklüfteten, nach Südosten gerichteten Felswand ca. $25 \mathrm{~m}$ über dem Wasserspiegel des Zabs, etwa $200 \mathrm{~m}$ nordöstlich einer Brücke, über die die Straße von Dokan nach Rania führt, angebracht $\left(36^{\circ} 12^{\prime} 55^{\prime \prime} \mathrm{N}, 4^{\circ} 59^{\circ} 18^{\prime \prime} \mathrm{O}, 520 \mathrm{~m}\right.$ ü NN). Es ist schwer zugänglich und für den Betrachter nicht einfach zu entdecken (Abb. 4-5).

Als erster interessierte sich Cecil J. Edmonds, britischer Officer und Beamter, für das Relief, der in den 1920er und 30er Jahren Kurdistan intensiv bereiste, und es in einem für ,The Geographical Journal' verfassten Beitrag erwähnte. ${ }^{5}$ In seinem Buch ,Kurds, Turks and Arabs' schilderte er die Ergebnisse seiner Recherchen, die er unternahm, nachdem er einmal einen Vermerk über ein assyrisches Relief am Darband-i Ramkan auf einer Karte gesehen hatte. ${ }^{6}$

3 Dazu Levine (1974, 9): „Not only is the Rania plain easily traversed, but the Kuh-i-Resh which marks its eastern border does not offer a barrier to further progress, as there is an easy track that runs along the river through Darband-i-Ramakhan"; s. auch Levine (1974, 13). 4 Wheildon Brown (1958); die aus Stein gemauerten Fortifikationen ließen sich nicht datieren.

5 Edmonds (1931, 351-354).

6 Edmonds (1957, 238-241, pl. 8b); in einem späteren Beitrag erwähnt er das Relief nur kurz (Edmonds 1966, 161). 


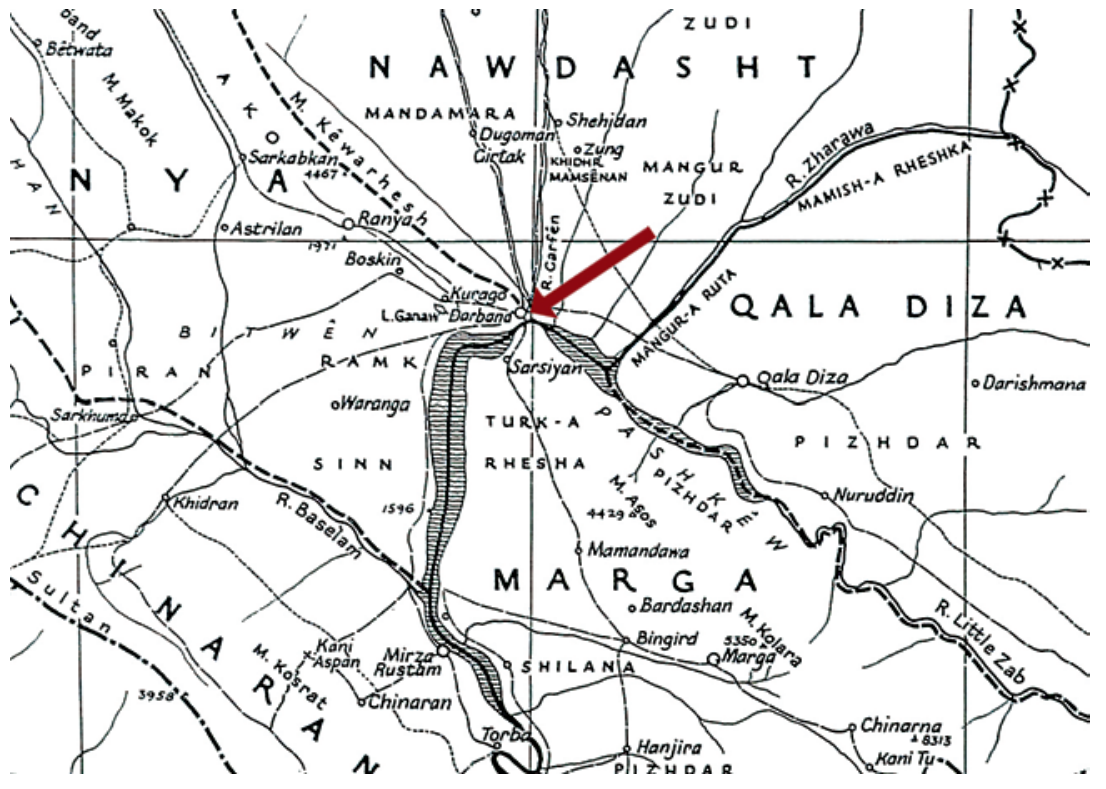

Abb. 1: Darband-i Ramkan auf der Karte von Edmonds (Edmonds 1957, Karte nach S. 262, Ausschnitt)

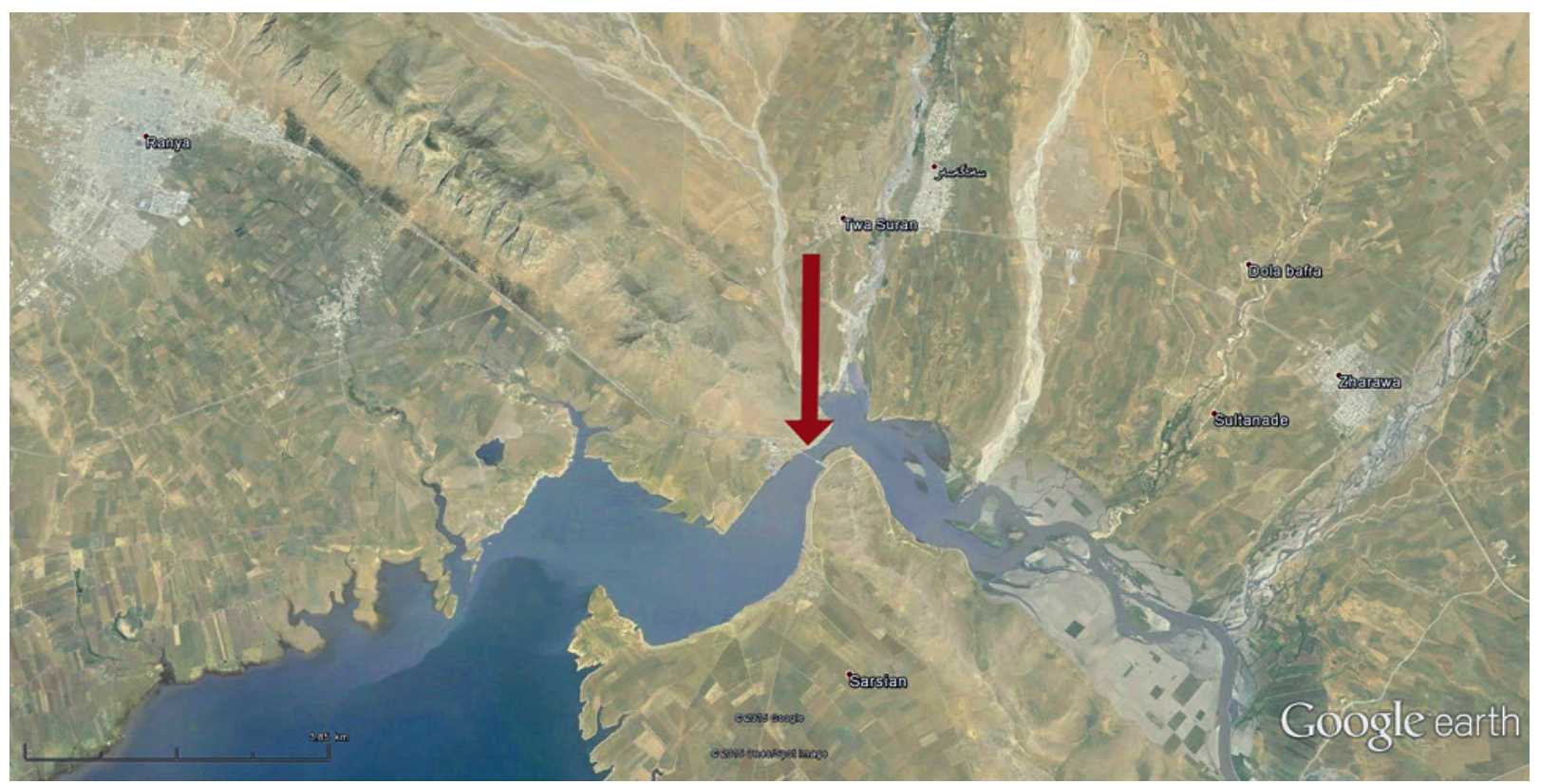

Abb. 2: Einmündung des Kleinen Zab in den Dokan-See (@ GoogleEarth)

„Local tradition still states that in ancient times the waters of the principal Ranya canal were carried across the Zab to the Marga Plain at Darband by means of an aqueduct, and the Kurds point out certain holes high up on the rock on the right bank as having held the chains suspending it. During my time there I never heard any mention of a carving or inscription at this point. My curiosity was therefore aroused some years later when I noticed in the map annexed to Sir Percy Sykes's larger History of Persia, precisely at the tip of the Darband hairpin, a bold red cross to indicate a 'most important Assyrian rock relief'” (Edmonds 1957, 238)
Edmonds bezog sich dabei auf eine Karte des zentralen Vorderasien („Map of Eastern Turkey in Asia, Syria and Western Persia - The Period of the Babylonian und Assyrian Empires"), die von der Royal Geographical Society 1910 veröffentlicht und von P. Sykes der ersten und zweiten Auflage seiner 'A History of Persia' 1915 und 1921 beigelegt wurde. ${ }^{7}$ Mit roten Kreuzen sind auf der Karte einige als

7 Die dritte, revidierte Auflage dieses Buches von 1958 enthält diese Karte nicht. 


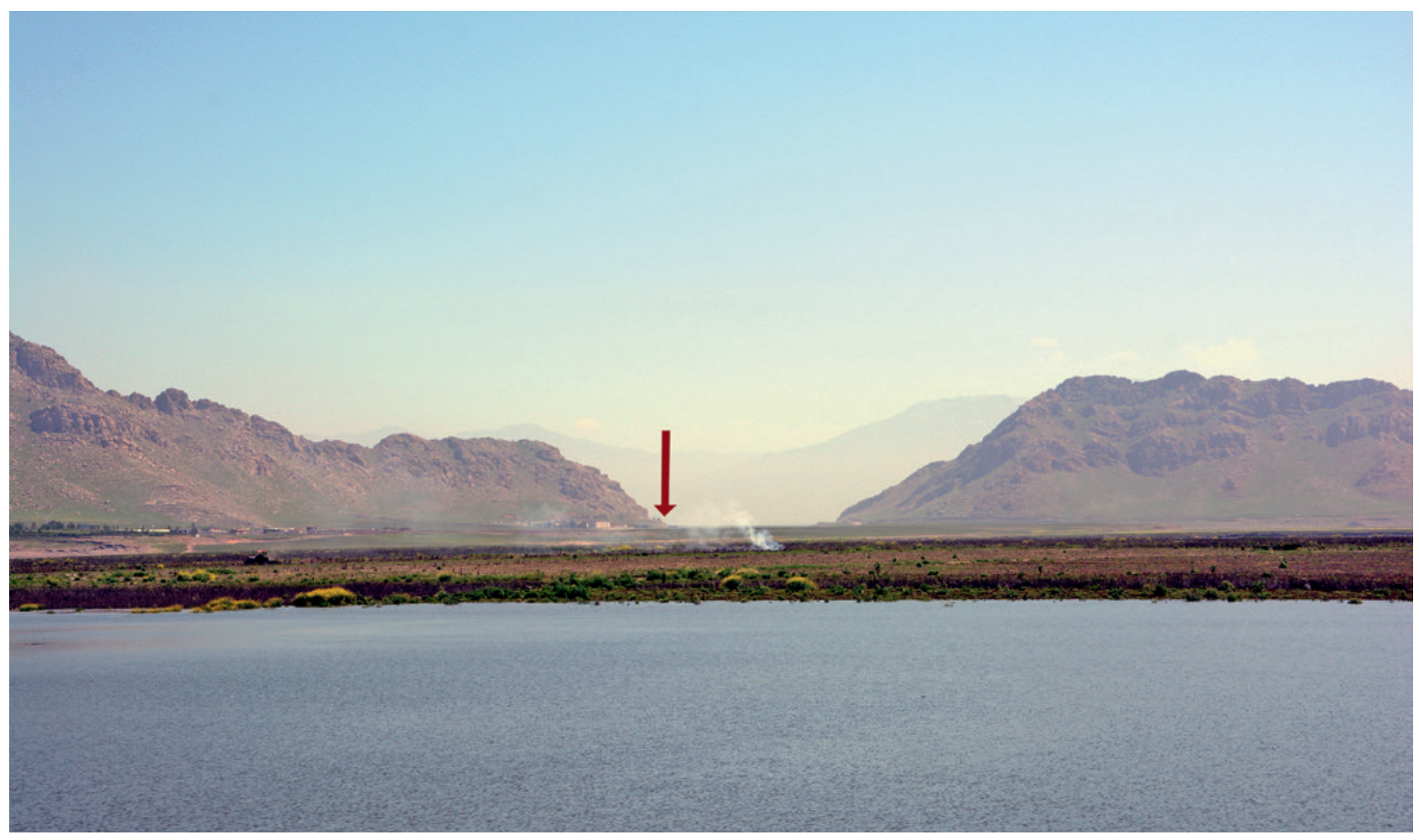

Abb. 3: Darband-i Ramkan (Darband-i Rania), Blick von Tell Šemšara (Foto: Verfasser, April 2015)

wichtig eingestufte 'assyrische' Felsinschriften markiert, nicht Felsreliefs wie bei Edmonds zu lesen ist. ${ }^{8}$ Immerhin folgte Edmonds diesem Hinweis. Nachdem er Berichte einiger Reisender zu dem Monument gelesen hatte, staunte er, wie unterschiedlich ihre Beobachtungen waren:

„The whole thing was getting 'curiouser and curiouser'. Clement apparently never saw or heard of a monument of any kind; Brzezowski saw a relief of a human figure but said categorically, 'il n'y a aucune inscription'; Maunsell saw a cuneiform inscription too high up on the rock to approach but saw no relief; I myself, with better opportunities than any of my three predecessors, had not seen, or even heard of any kind of carving at Darband itself.” (Edmonds 1957, 240).

Um seine gesteigerte Neugier zu befriedigen, beschloss Edmonds schließlich, das Relief persönlich zu erkunden:

„I made an opportunity in the autumn of the same year, 1931, to visit Darband. Guided by them, and in spite of a painful attack of lumbago, I managed to climb up to the place, which was exactly as Maunsell had shown it in his sketch. There was a panel smoothed in the face of the rock, measuring about three feet long and two and a half feet high; in the left half, as one looked at it, there was a slight swelling (one could hardly call it more)

8 Die Markierung auf der Karte befindet sich südlich des Kleinen Zab. Es ist deshalb nicht auszuschließen, dass sie sich auf ein anderes Denkmal bezieht. which might once have been a human figure in relief, but there was no sign of any cuneiform inscription. It was not possible to photograph the panel from directly in front, but the accompanying picture of Mamand Agha shows the left end of it to the right of his head. When I returned to the road below, knowing where to look, I could see the end of the panel with the swelling quite distinctly." (Edmonds 1957, 240 f.). ${ }^{9}$

Die nächste Erwähnung findet sich bei Jørgen Læssøe, der anlässlich der Veröffentlichung des in Tell Šemšara (Šušarrā) ausgegrabenen Keilschriftarchivs Informationen zur Rania-Ebene und ihrer Umgebung zusammenstellte. Offensichtlich hatte er das Relief selbst nicht gesehen und charakterisierte es anhand eines Berichts, den er von T. Wheildon Brown ${ }^{10}$ erhalten hatte:

"The existence of another relief at Darband-i-Ramkan, where the Lesser Zab leaves the Nawdasht Valley at Sungasur and enters the Plain south of Rania, has long been suspected in spite of conflicting reports by various travelers (...). The relief, a panel ab. one metre wide and $60 \mathrm{cms}$. high, was observed by Mr. T. Wheildon Brown, in June, 1958, during the visit to the Sungasur Gorge. The carving is ab. 20 metres above the ground, on the steep rock face which is separated from the right bank of the Lesser Zab only

9 Auf dem genannten Foto von Mamand Agha (Edmonds 1957, ill. 8b) ist vom Relief wenig erkennbar.

10 Wheildon Brown war laut Læssøe (1959, 7), Senior Assistant to the Resident Engineer in Dokan; s. auch Levine (1974, 3 Anm. 9). 


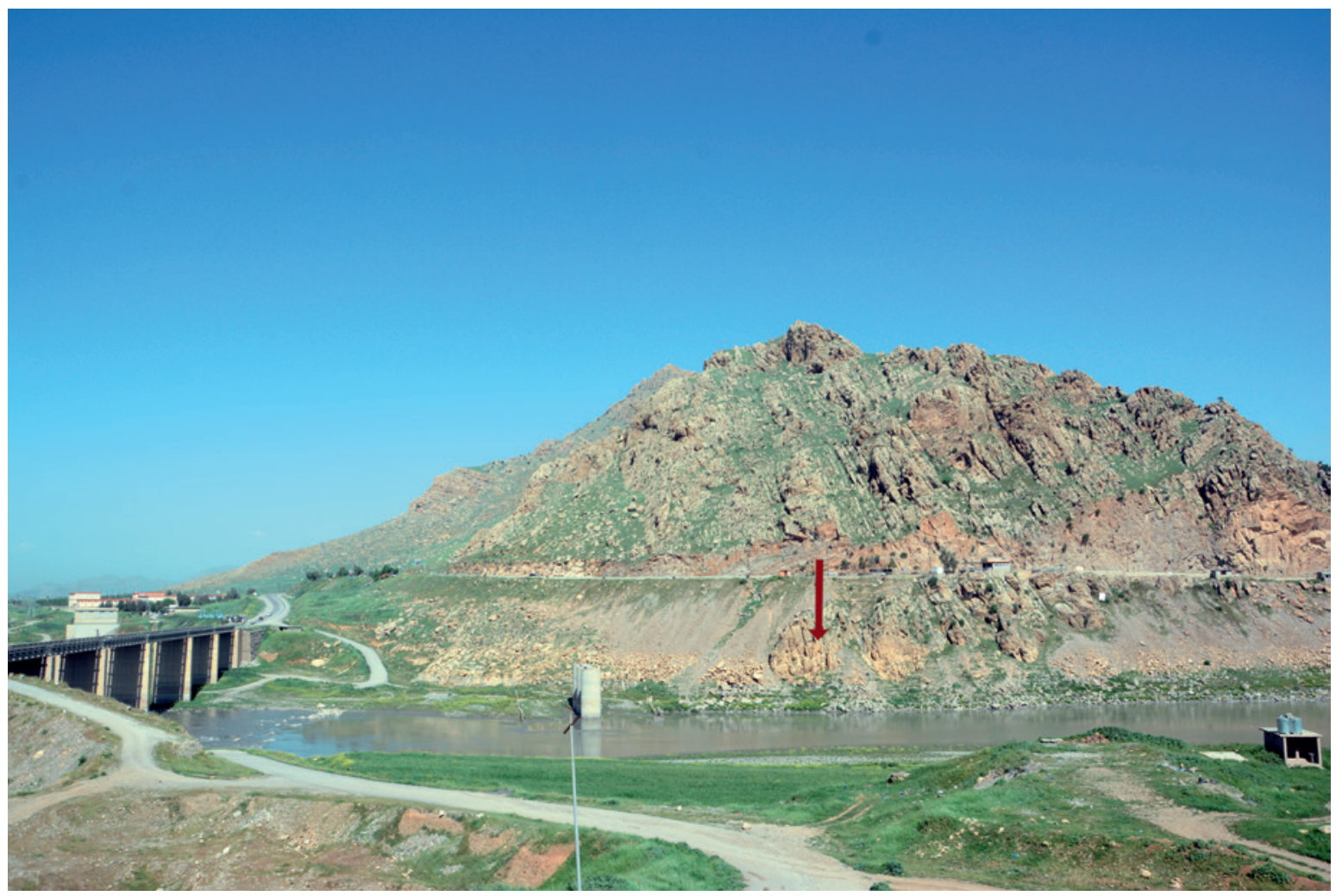

Abb. 4: Darband-i Ramkan, Blick vom südlichen Ufer des Kleinen Zab, links Brücke und Straße nach Rania (Foto: Verfasser, April 2015)

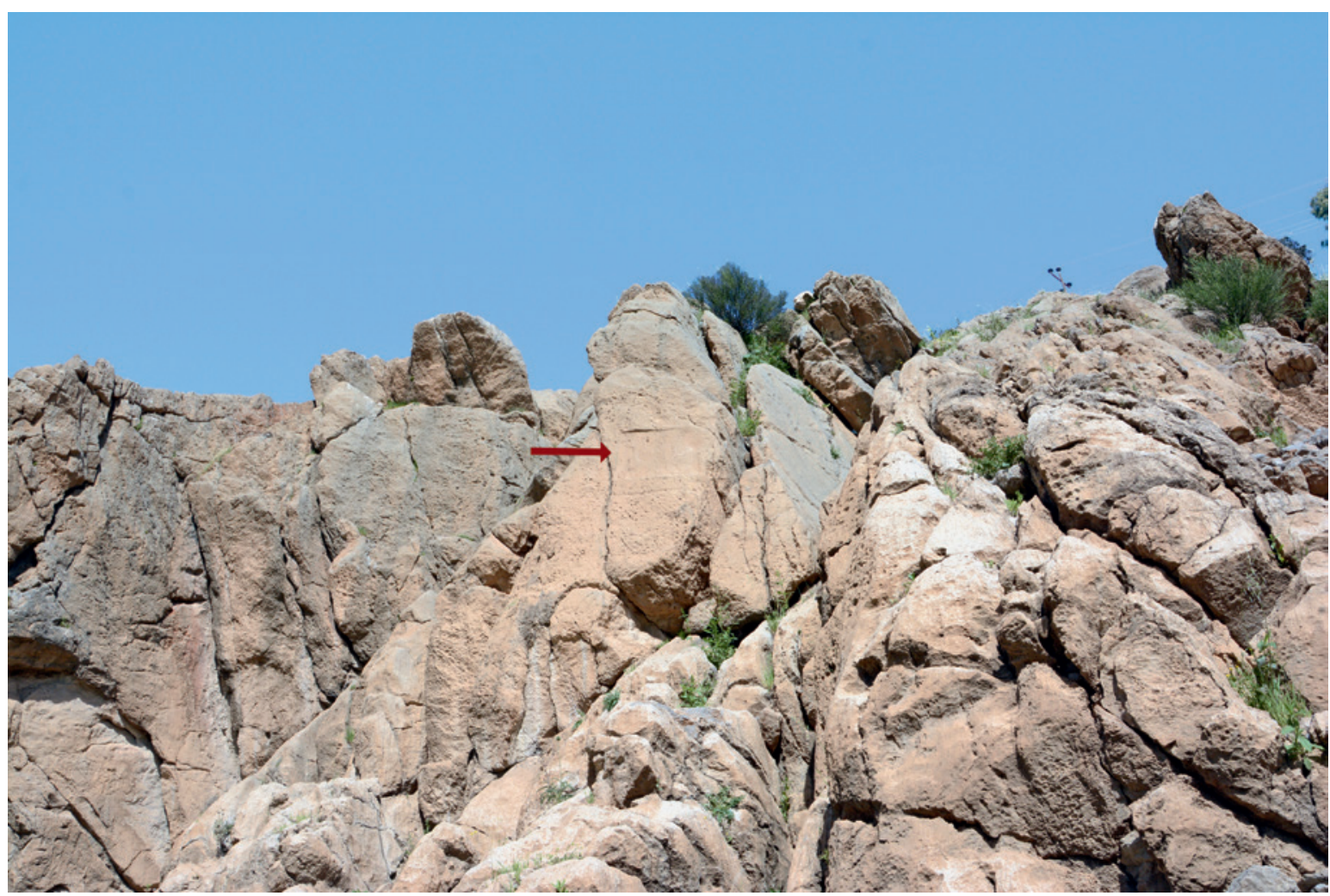

Abb. 5: Relief vom Niveau des Flusses aus aufgenommen (Foto: Verfasser, April 2015) 
by a narrow track. From Mr. Wheildon Brown's description of the relief, which he kindly communicated to me in a letter dated the $3^{\text {rd }}$ July, 1958, it would appear very likely that this is another specimen of the Naram-Sīn "Victory Stela" type, and in fact very similar to the relief at Darband-i-Gawr.” (Læssøe 1959, 14 f.).

Kurz darauf wurde das Relief von Kazem Al-Ǧenabi veröffentlicht. ${ }^{11}$ Es ist unklar, ob er es tatsächlich gesehen hat, weil er seinen eigenen Angaben zufolge den Bericht anhand einer Beschreibung und Fotoaufnahme von Akram Shukri vom 7. Oktober 1960 verfasste. Laut seiner Schilderung ist das Bild $130 \mathrm{~cm}$ breit, $80 \mathrm{~cm}$ hoch und $4 \mathrm{~cm}$ tief. Die mit einer perspektivischen Verzerrung gezeichnete Skizze zeigt auf der linken Seite ein längliches Dreieck, das ein nach hinten ausgestrecktes Bein darstellen könnte, und auf der rechten Seite eine Figur, die AlǦenabi für die Göttin Ištar hielt (Abb. 6). Rechts von ihr, an der oberen Kante des Relieffeldes, sollte sich ein Kreis befinden, den er als Symbol der Göttin zu erklären versuchte. Al-Ǧenabi verglich die Darstellung mit dem Felsrelief Anubaninis in Sar-i Pol-i Zohab (Relief II).

Den Aufsatz von Al-Ǧenabi nutzte anschließend Jutta Börker-Klähn für die folgende Beschreibung:

„Vom Bild ist so gut wie nichts erhalten. Die Umzeichnung zeigt links einen undeutbaren Reliefrest, rechts Fragmente einer menschlichen Figur(?) und ganz oben rechts - also an sehr ungewöhnlicher Stelle - das Mond-/Sonnen-Symbol.“ (BörkerKlähn 1982, 140). ${ }^{12}$

Ein anderes Bild sah Simone Mühl während ihres Besuches bei Darband-i Ramkan. Sie merkte an, es handele sich um einen siegreichen Herrscher, der über einem besiegten Feind stehend eine Waffe, vermutlich Keule, in der Hand schwinge (Mühl 2013, 145f. Anm. 890).

Im April dieses Jahres nutzte der Autor während eines Ausflugs nach Rania die Gelegenheit, das Denkmal zu besichtigen, ${ }^{13}$ und konnte feststellen, dass die Beobachtungen von Edmonds und Mühl richtig waren. Die Darstellung zeigt auf der rechten Seite des Relieffeldes eine menschliche Figur in charakteristischer Siegerpose über einen liegenden Feind (Abb. 7-8). Der Sieger ist in aktiver Kampfhaltung abgebildet: mit seinem hoch nach hinten erhobenen rechten Arm holt er zum Schlag gegen einen gestürzten Gegner aus und erinnert damit an den triumphierenden Krieger vom oberen Register der Dāduša-Stele

11 Al-Ǧenabi (1962, 203f. Taf. 1-2) Abb. 2 (Foto des Felsens) und Abb. 3 (Umzeichnung).

12 Börker-Klähn (1982, 140); der Beitrag von Al-Ǧenabi ist dort mit einer falschen Seitenangabe zitiert.

13 Bei Ulrike Bürger und Shwenawar Barzan, die mich begleiteten, bedanke mich an dieser Stelle für ihre Hilfe.

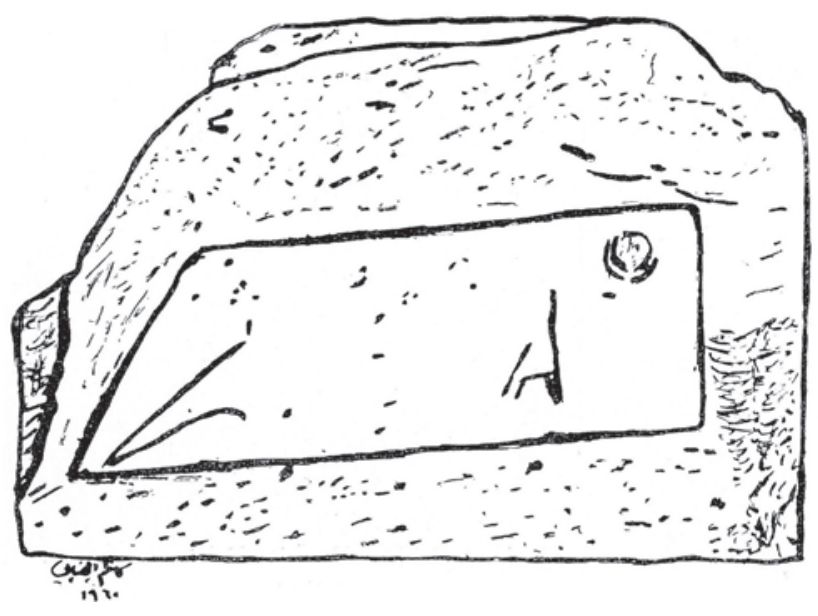

Abb. 6: Umzeichnung des Reliefs vom 1960 (Al-Ǧenabi 1962, Abb. 3 nach S. 204)

(Abb.9). In der Hand hält er eine Waffe, deren Schaft am linken Rand des Relieffeldes beginnt und in einer nach oben abgerundeten Verdickung zwischen seinem Kopf und dem oberen Bildrand endet. Bei der Waffe handelt es sich höchstwahrscheinlich um eine Fensteraxt, mit der die triumphierenden Fürsten von Sar-i Pol-i Zohab und Bitwāta bewaffnet sind. Den linken Oberarm kann man nur noch an der Innenkante erkennen; sie führt vor seiner Brust schräg nach unten und verliert sich an der Stelle, wo man den Ellbogen erwartet. Dort könnte der Arm nach vorne angewinkelt sein, in Richtung einer schwachen, schräg nach unten verlaufenden Linie. Diese dürfte man mit Vorsicht als Teil einer Lanze interpretieren, mit welcher der Krieger, ähnlich wie Šamšī-Adad I. auf der Mardin-Stele, auf den liegenden Feind zielte. ${ }^{14}$ Man kann jedoch nicht ausschließen, dass er mit der linken Hand nach dem Arm

14 Šamši-Adad ist mit Schaftlochaxt und Lanze bewaffnet wie die Krieger im zweiten Register der Daduša-Stele. Börker-Klähn (1982, 165) hielt die Lanze für ein Seil („Der Fürst reißt an einem dicken Seil einen Feind vom Boden, tritt ihn und bedrängt ihn mit der Kriegsaxt“) und ergänzte ihre Umzeichnung des Reliefs um einen nicht existierenden Nasenring, worauf bereits Seidl (1986, 321) hingewiesen hat. Braun-Holzinger $(2007,154)$ wiederholte diese Deutung („In der linken Hand hält der Sieger das Seil, das zur Nase des Besiegten führt, wie auf dem Šuilija-Siegel“). Ein Seil in der Hand des Triumphators (Herrscher oder Gottheit) ist von anderen derartigen Darstellungen jedoch nicht bekannt. In den Triumphszenen werden Gefangene am Seil von begleitenden Gottheiten gehalten, während der siegreiche Herrscher mit Waffe in herabhängender Hand gezeigt wird. Den besiegten Feind an einem Seil zu ziehen und gleichzeitig mit einer Waffe anzugreifen, wäre ungewöhnlich und auch kaum mit einer Kampfsituation vereinbar. Vgl. auch Beschreibungen bei Seidl (1975, 301 Nr. 182); Miglus (2003, 405; 2008, 232); B. André-Salvini in: Marzahn/Schauerte $(2008,130)$. 


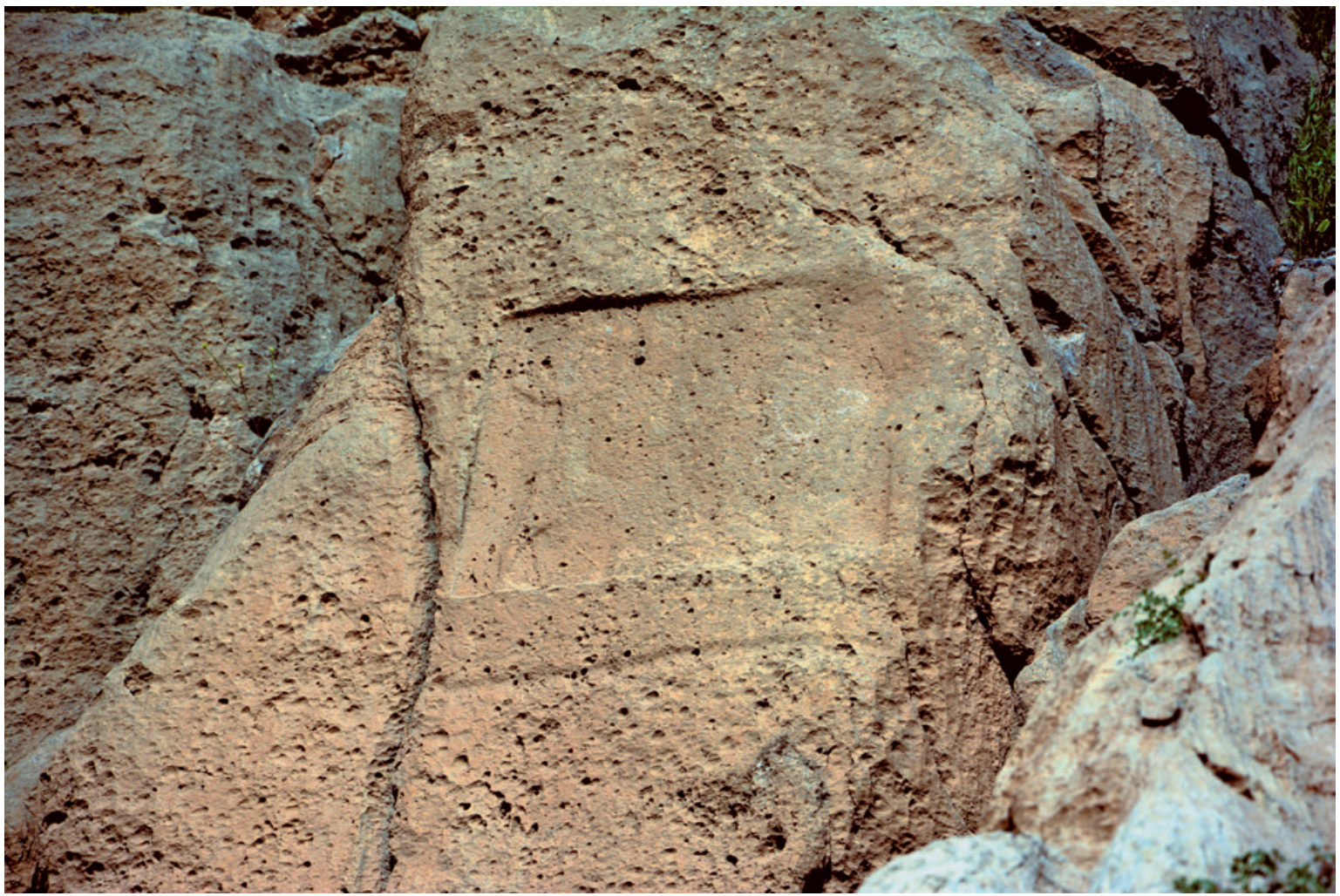

Abb.7: Relief von Südosten, von unten aus einer Entfernung von ca. 4 m aufgenommen (Foto: U. Bürger, April 2015)

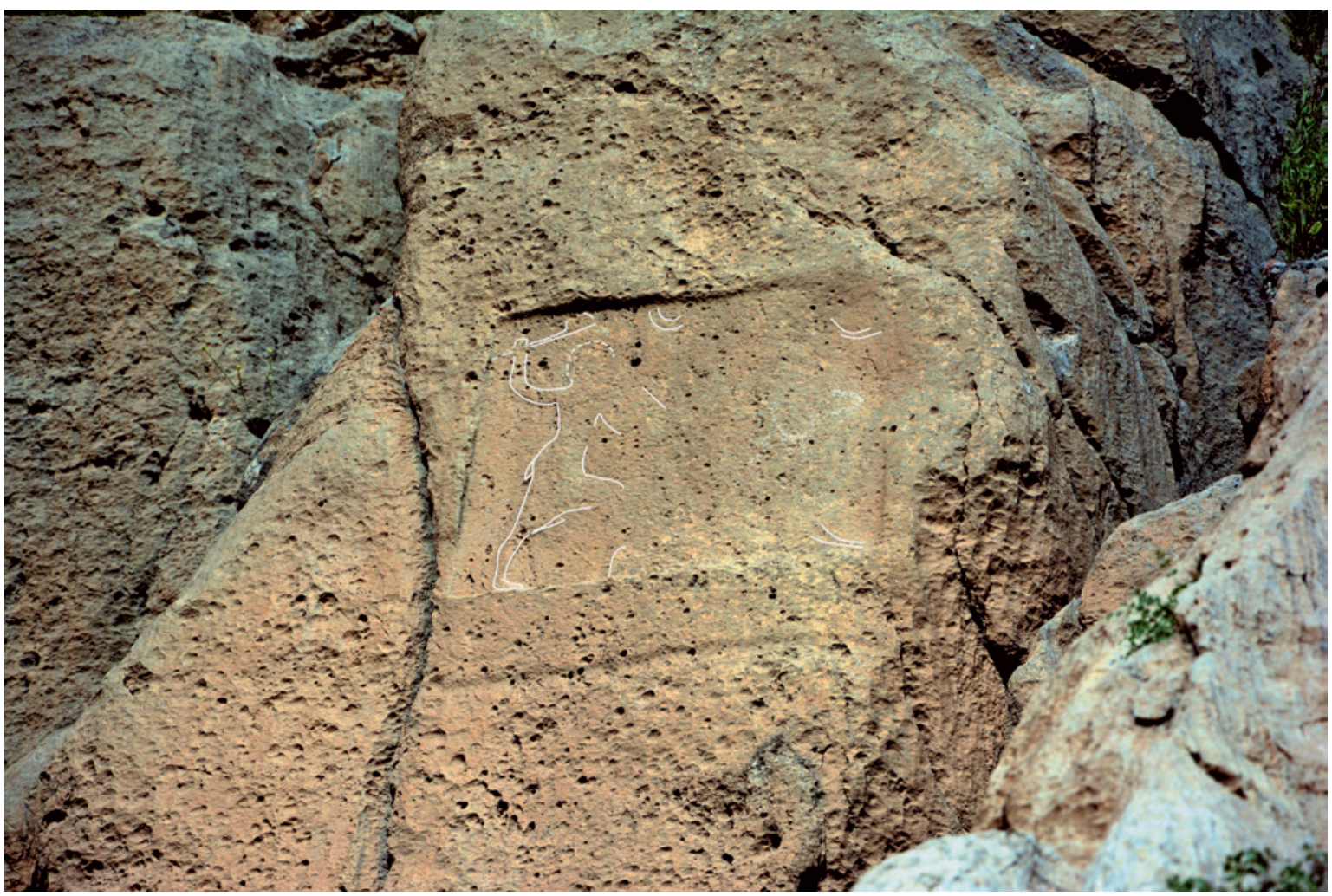

Abb. 8: Relief mit Umrissen der sichtbaren Reste (Foto: U. Bürger/Verfasser, April 2015) 


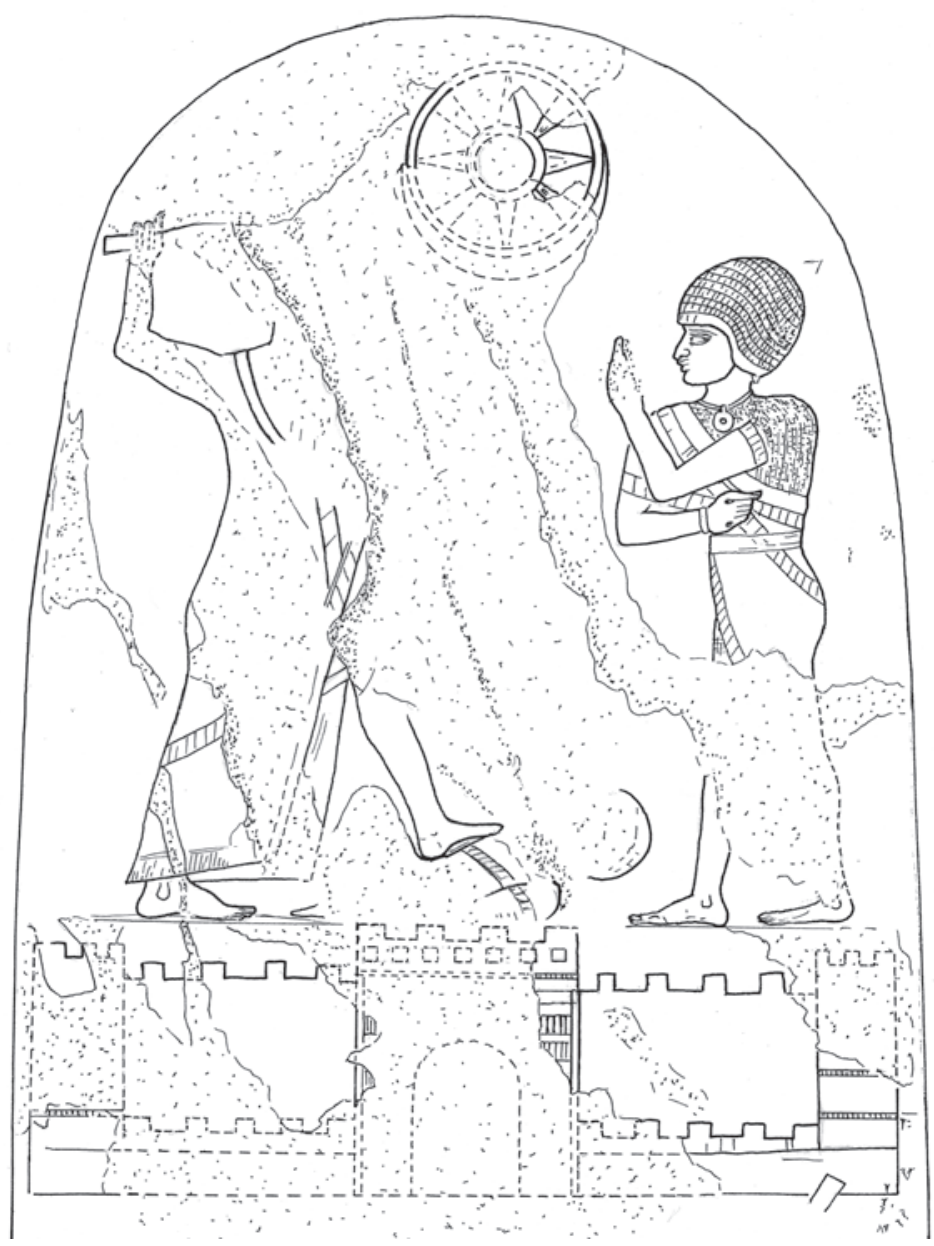

Abb. 9: Triumphator-Darstellung auf der Siegesstele des Königs Dāduša von Ešnunna (Miglus 2003, 415 Abb. 9) des besiegten Feindes griff, was verschiedene Darstellungen des ,Gottkönigs als Krieger ‘ in der Isin-Larsa-zeitlichen und altbabylonischen Glyptik und ein Terrakottarelief aus Tell Ḥarmal (Abb. 10) belegen. Die Kopfbedeckung ist nicht erkennbar, ja nicht mal der Umriss des Kopfes lässt sich sicher rekonstruieren. Der Oberkörper verjüngt sich leicht bis zur Taille, an der vermutlich das Kriegsgewand, ein Wickelrock, beginnt, dessen unterer Abschluss sich deutlich in Kniehöhe abzeichnet. Ein Gürtel bzw. die obere Stoffkante ist jedoch ebenso wenig zu erkennen wie andere Details, die von ähnlichen Darstellungen bekannt sind. Lediglich am Gesäß über dem rechten, nach hinten ausgestreckten Bein ist ein kleiner, herabhängender Zipfel des Gewandes sichtbar, ähnlich denen, die man von den Reliefs von Darband-i Gaur, Sar-i Pol I und II und Bitwāta kennt ${ }^{15}$. Die rechte Wade und der Fuß sowie der linke, nach vorn ausgestreckte Oberschenkel sind im Umriss erhalten. Der linke Fuß, mit dem der Sieger den Besieg-

15 Beschreibung des Gewandes von Darband-i Gaur und Bitwāta siehe Strommenger $(1963,86)$ und Shaffer/Wasserman/Seidl (2003, 42). ten niedertritt, ist nicht mehr zu erkennen. Von dem liegenden Feind gibt es nur undeutliche Spuren, von denen nur das im Knie abgeknickte Bein etwas stärker hervortritt. Auf der rechten Seite, wo die Zeichnung Al-Ǧenabis eine stehende Person zeigt, ist kaum etwas zu erkennen; lediglich eine leicht verdickte Fläche am oberen Bildrand könnte man für eine Spur eines Kopfes halten. Die Stelle in der rechten oberen Ecke, wo Al-Ǧenabi den Ištar-Stern sah, den Börker-Klähn für das Sonne-Mond-Symbol hielt, ist $\mathrm{zu}$ stark verwaschen, um diesbezügliche Aussagen zu erlauben. Eine doppelte Scheibe oder Sonnenscheibe mit darunter befindlicher Mondsichel scheint sich hingegen schwach links von der Mittelachse des Bildes in der Nähe des Kopfes des Triumphators abzuzeichnen. Unter der Bildfläche befindet sich ein länglicher, ca. 13-14 cm hoher Streifen, der unten von einer breit eingemeißelten Rille begrenzt ist. Ob es sich dabei um ein Feld für eine Inschrift handeln könnte, ist schwer zu sagen: Weder hier noch im Bildhintergrund sind Spuren von Schriftzeichen zu sehen. Womöglich kann dieser Streifen als Standfläche gedeutet werden, die im Relief Sar-i Pol I über der Inschrift positioniert ist. 


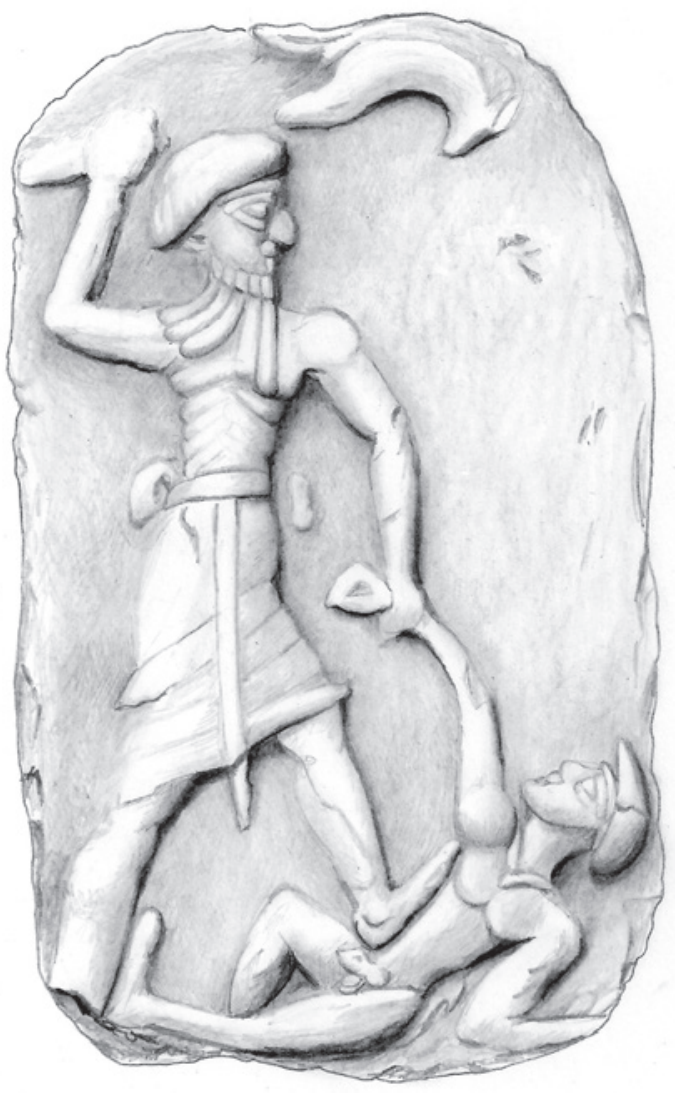

Abb. 10: Terrakottarelief IM 54631 aus Tell Ḥarmal mit Darstellung des triumphierenden „Gottkönigs als Krieger“ (Bürger/Miglus, in Vorb.)

Die bisherigen vagen Deutungen des Felsreliefs an der Schlucht Darband-i Ramkan gingen davon aus, dass es einen siegreichen Herrscher darstellt, ähnlich wie auf den Felsreliefs von Sar-i Pol-i Zohab oder Darband-i Gaur. Auf der rechten Seite wäre genug Platz für eine (weibliche) Gottheit, die ihm zum Sieg verhalf. Das Erhaltene erlaubt jedoch auch eine andere Interpretation. Das Motiv des mit hocherhobener Waffe kämpfenden Triumphators, der den Feind niedertritt, war in der altmesopotamischen Kunst siegreichen Gottheiten vorbehalten. Dies belegen Siegeldarstellungen mit dem kämpfenden ,Gottkönig als Krieger', während triumphierende Fürsten in der Regel nicht in dieser Pose gezeigt wurden. ${ }^{16}$ Der Sieger von Darband-i Ramkan müsste demnach als kriegerischer Gott gedeutet werden, und auf der rechten, zerstörten Seite des Bildes dürfte man ähnlich wie im oberen Register der Daduša-Stele den vor dem Gott stehenden Auftraggeber

16 Dazu Braun-Holzinger (2007, 153): „Der eine Waffe schwingend erhobene rechte Arm ist nie bei einem kriegerischen Herrscher belegt, jedoch die übliche Haltung des mythischen Triumphators, der in altbabylonischer Zeit beliebt ist, und auch die Ikonographie des Wettergottes prägt“. des Reliefs ergänzen. ${ }^{17}$ Die Deutung der Hauptszene auf der Dāduša-Stele ist jedoch auch nicht unumstritten. ${ }^{18}$

Möglicherweise lassen sich bei günstigen Lichtverhältnissen noch weitere Details des Felsreliefs erschließen. ${ }^{19}$ Die beschriebenen Reste reichen jedoch bereits aus, um es zeitlich in das 19.-18. Jahrhundert v. Chr. einzuordnen. Das Motiv der mit der Waffe über dem Kopf kämpfenden Gottheit ('smiting god') wurde zum festen, vor allem in der Glyptik beliebten Bestandteil der mesopotamischen Ikonographie der Larsa-Zeit. ${ }^{20}$ Typisch ist der ,Gottkönig als Krieger' in kurzem Rock und mit erhobenem Sichelschwert, der eine Hand des Besiegten ergreift und auf ihn tritt. Auch das oben erwähnte Terrakottarelief aus Tell Harmal (Abb. 10), auf dem der Triumphator seinen Fuß auf den liegenden Gegner gestellt hat und ihn mit erhobenem Krummschwert bedroht, datiert kurz vor Hammurapi. ${ }^{21}$ Das Relief von Darband-i Ramkan dürfte deshalb mindestens ein Jahrhundert jünger sein als die Felsreliefs von Darband-i Gaur, Sar-i Pol-i Zohab I-IV und Bitwāta, die einen Triumphator mit Bogen und herabhängender Fensteraxt darstellen und im 21.-20. Jahrhundert v. Chr. entstanden sind (Seidl in: Shaffer/Wasserman/Seidl 2003, 45. 49-52). Es wäre verlockend, das Bild in die Zeit des großen Kräftemessens zu datieren, als Daduša, Šamšì-Adad und Išme-Dagan Kriege in dieser Region führten, und es einem dieser Herrscher zuzuschreiben. In Frage käme auch einer der Fürsten der Turukkäer, die damals zeitweise die RaniaEbene und die nahe der Schlucht gelegene Stadt Šušarrā (Tell Šemšara) kontrollierten (dazu s. Eidem 2011-13 und Ziegler 2014).

17 In der Glyptik kann auch eine zweite Gottheit vor dem ,Gottkönig als Krieger' stehen. Bei Boehmer in: Orthmann (1975, 347 f. Abb. 103b) ist das die kriegerische Ištar im Schlitzmantel mit nach unten herabhängendem Sichelschwert, die ihren Fuß auf einen Löwen setzt (Zeit des Apil-Sîn von Babylon).

18 Miglus $(2003,403)$ und Braun-Holzinger (2007, 153f.) halten den Triumphator auf der Dāduša-Stele für den Wettergott, Seidl (2003, 45f. und 2011-13, 136), Charpin (2004, 157-XXX) sowie Uehlinger (2008, 534f.), dem Wortlaut der Inschrift folgend, sind hingegen der Meinung, dass es sich dabei um den siegreichen König Dāduša handeln muss.

19 Der Autor besichtigte das Denkmal am 19. 04. 2015 zwischen 9 und $10 \mathrm{Uhr}$, als die Sonne schräg von der rechten Seite auf das Bild fiel. Am späten Nachmittag befindet es sich allerdings im Schatten des Felsens.

20 Collon (1986, 165 f. Pl. XXXI Nr. 418-424: „This gesture of victory is not found in Near Eastern iconography until it was adopted in the 19th century B. C., presumably via Syria and Anatolia"); Blocher (1992, 125f. Abb. 58-59). Ähnliche Posen erscheinen jedoch schon in den Götterkämpfen auf den akkadischen Siegeln: Miglus (2008, 232). 21 IM 54631: Opificius (1961, 134 Taf. 12 Nr. 480); Miglus (2003, 418, Abb. 17. 1). Es wurde in Schicht II, einem Horizont der HammurapiZeit, in der Nachbarschaft des sog. Serai-Gebäudes gefunden. 


\section{Literatur}

Al-Ǧenabi, K. (1962): Manzuta Darband-i Ramkan, Sumer 18, 203-204

Blocher, F. (1992): Siegelabrollungen auf frühaltbabylonischen Tontafeln im British Museum. Ein Katalog. Münchner Vorderasiatische Studien 10. München/Wien

Börker-Klähn, J. (1982): Altvorderasiatische Bildstelen und vergleichbare Felsreliefs. BaF 4. Mainz am Rhein

Braun-Holzinger, E. A. (2007): Das Herrscherbild in Mesopotamien und Elam. AOAT 342. Münster

Bürger, U./ P. A. Miglus (in Vorb.): Tall Ḥarmal - Šaduppûm I: Architektur und Kleinfunde

Charpin, D. (2004): Chroniques Bibliographiques. 3. Données nouvelles sur la région du Petit Zab au XVIIIe siècle av. J.-C., RA 98, 151-178

Collon, D. (1986): Catalogue of the Western Asiatic seals in the British Museum. Cylinder seals III: Isin-Larsa and Old Babylonian periods, British Museum Press. London

Edmonds, C. J. (1931): A third note on rock monuments in Southern Kurdistan, The Geographical Journal, Vol. 77, No. 4, 350-355

Edmonds, C. J. (1957): Kurds, Turks and Arabs. Politics, travel and research in North-Eastern Iraq 1919-1925. London

Edmonds, C. J. (1966): Some ancient monuments on the IraqiPersian boundary, Iraq 28, 159-163

Eidem, J. (2011-13): Šušarrā. Ancient town in N-E Iraq, in: RIA 13, 360-362

Læssøe, J. (1959): The Shemshāra tablets. A preliminary report. Archaeologisk-kunsthistoriske Meddelelser. Det Kongelige Danske Videnskabernes Selskab 4 no. 3. København

Levine, L. D. (1974): Geographical studies in the Neo-Assyrian Zagros. Toronto/London

Marzahn J./G. Schauerte (Hrsg.), (2008): Babylon: Wahrheit. Eine Ausstellung des Vorderasiatischen Museums Staatliche Museen zu Berlin. München

Miglus, P. A. (2003): Die Siegesstele des Königs Dāduša von Ešnunna und ihre Stellung in der Kunst Mesopotamiens und der Nachbargebiete, in: R. Dittmann [u. a.] (Hrsg.), Altertumswissenschaften im Dialog. Festschrift für Wolfram Nagel. AOAT 306. Münster, 397-419
Miglus, P. A. (2008): Kings go into battle. Representations of the Mesopotamian ruler as a warrior, in: Ph. Abrahami/L. Battini (Hrsg.), Les armées du Proche-Orient ancient (IIle - ler mill. av. J.-C.). BAR Int. Ser. 1855. Oxford, 231-246

Mofidi Nasrabadi, B. (2004): Beobachtungen zum Felsrelief Anubaninis, ZA 94, 291-303

Mortensen, P. (1970): Tell Shimshara. The Hassuna Period. København

Mühl, S. (2013): Siedlungsgeschichte im mittleren Osttigrisgebiet. Vom Neolithikum bis in die neuassyrische Zeit. ADOG 28. Wiesbaden

Opificius, R. (1961): Das altbabylonische Terrakottarelief. Berlin

Orthmann, W. (Hrsg.) (1975): Der Alte Orient. Propyläen Kunstgeschichte 14. Berlin

Postgate, J. N./M. D. Roaf (1997): The Shaikhan relief, Al-Rāfidān 18, 143-160

Shaffer, A./N. Wasserman/U. Seidl (2003): Iddi(n)-Sîn, king of Simurrum: A new rock-relief inscription and a reverential seal, ZA 93, 1-52

Seidl, U. (1975): Babylonische und assyrische Flachbildkunst des 2. Jahrtausends v. Chr., in: Orthmann 1975, 298-309

Seidl, U. (1986): Buchbesprechung von J. Börker-Klähn 1982, Orientalia 55, 320-327

Seidl, U. (2011-13): Stele. A. II. In Mesopotamien. Archäologisch, in: RIA 13, 133-137

Sykes, P. (1915; 1917): A History of Persia. London

Strommenger, E. (1963): Das Felsrelief von Darband-i Gaur, BagM 2, 83-88

Uehlinger, Ch. (2008): Gott oder König? Bild und Text der altbabylonischen Siegesstele des Königs Dāduša von Ešnunna, in: M. Bauks/K. Liess/P. Riede (Hrsg.), Was ist der Mensch, dass Du seiner gedenkst? (Psalm 8,5). Aspekte einer theologischen Anthropologie. Festschrift für Bernd Janowski zum 65. Geburtstag. Neukirchen-Vluyn, 515-538

Wheildon Brown, T. (1958): A report on the discovery of a line of ancient fortifications on a ridge to the east of the Rania plain, Sulaimaniyah liwa, Sumer 14, 122-124

Ziegler, N. (2014): Turukkû, Turukkäer, in: RIA 14, 209-212 